\title{
Determinants of peak bone mass and bone mineralization rates in 470 healthy children, adolescents and young adults: A prospective study
}

\author{
Magnar Gunnes, M.D. \\ Senter for klinisk osteoporoseforskning, Nygårdsveien 6, 5500 Haugesund
}

Increasing peak bone mass (PBM) and slowing bone loss are generally accepted as preventive measures against osteoporotic fractures later in life. As osteoporosis treatment do not restore bone mass and bone architecture, increasing PBM or preventing bone loss leave the best options of disease handling. While postmenopausal bone loss has been studied for years, knowledge about influences on PBM are scarce.

Hereditary factors account for about $80 \%$ of PBM variance. About $20 \%$ is due to environmental factors which can be modified favourably to increase PBM. These factors are presently poorly understood. We therefore investigated forearm trabecular and cortical bone mass by single photon absorptiometry, diet, exercise and daylight exposure in 470 boys and girls in 1992. A follow-up was done in 1993. The subjects were 8.2-17.2 years of age at baseline.

Bone mineral density (BMD) increased heterogeneously according to bone type, age, sex and environmental influences. While girls achieved adult premenopausal BMD values between 14 and 17 years of age, boys appeared to increase bone density beyond these ages. Exercise was the most important environ- mental influence on BMD accretion rates. The active group ( $>11$ hours exercise/week) increased their BMD by $14-128 \%$ in excess of the increase of the BMD of the sedentary group $(<4$ hours exercise/week). There was a significant interaction between calcium intake and exercise on BMD accretion rates in children $<11$ years of age. In children equal to or older than 11 years this activity/calcium intake term approached a significant value. Intake of polyunsaturated fat was positively associated with BMD accretion rate in girls $\geq 11$ years of age, while sodium intake was negatively associated with BMD accretion rate in girls $<11$ years of age. Daylight exposure had no effect on the BMD increase rate.

Our findings indicate that physical activity should be encouraged in order to optimize PBM. The greater effect on bone mass by physical activity when combined with a high calcium intake ( $>800 \mathrm{mg} /$ day) should be taken advantage of when giving health recommendation to young people. Furthermore, our results indicate that sodium should be restricted while polyunsaturated fat should be encouraged in order to enhance PBM. 